**TITLE**

ASP Conference Series, Vol. **VOLUME**, **PUBLICATION YEAR **

**EDITORS ${ }^{* *}$

\title{
Cosmological Parameters and Hyper-Parameters: The Hubble Constant from Boomerang and Maxima
}

\author{
Ofer Lahav \\ Institute of Astronomy, Madingley Road, Cambridge CB3 OHA, UK
}

\begin{abstract}
We generalise the procedure for joint estimation of cosmological parameters to allow freedom in the relative weights of various probes. This is done by including in the joint Likelihood function a set of 'Hyper-Parameters', which are dealt with using Bayesian considerations. The resulting algorithm is simple to implement. We illustrate the method by estimating the Hubble constant $H_{0}$ from the recent Cosmic Microwave Background experiments Boomerang and Maxima. For an assumed flat $\Lambda$-CDM model with fixed parameters $\left(n=1, \Omega_{m}=\right.$ $\left.1-\lambda=0.3, \Omega_{b} h^{2}=0.03, Q_{\mathrm{rms}}=18 \mu K\right)$ we solve for a single parameter, $H_{0}=79 \pm 4 \mathrm{~km} / \mathrm{sec} / \mathrm{Mpc}(95 \% \mathrm{CL}$, random errors only), slightly higher but still consistent with recent results from Cepheids. We discuss how the 'Hyper-Parameters' approach can be generalised for a combination of cosmic probes, and for other priors on the Hyper-Parameters.
\end{abstract}

\section{Introduction}

Several groups (e.g. Gawiser \& Silk 1998; Webster et al. 1998; Lineweaver 1998; Eisenstein, Hu \& Tegmark 1999; Efstathiou et al. 1999; Bridle et al. 1999, 2000; Bahcall et al. 1999) have recently discussed the estimation of cosmological parameters by joint analysis of data sets, e.g. Cosmic Microwave Background (CMB), SNe Ia, redshift surveys, cluster abundance and peculiar velocities.

While joint Likelihood analyses employing both CMB and LSS data are allowing more accurate estimates of cosmological parameters, they involve various subtle statistical issues:

- The choice of the model parameter space is somewhat arbitrary.

- One commonly solves for the probability for the data given a model (e.g. using a Likelihood function), while in the Bayesian framework this should be modified by the prior for the model.

- If one is interested in a small set of parameters, should one marginalise over all the remaining parameters, rather than fix them at certain (somewhat ad-hoc) values ?

- The 'topology' of the Likelihood contours may not be simple. It is helpful when the Likelihood contours of different probes 'cross' each other to yield a global maximum (e.g. in the case of $\mathrm{CMB}$ and $\mathrm{SNe}$ ), but in other 
cases they may yield distinct separate 'mountains', and the joint maximum Likelihood may lie in a 'valley'.

- Different probes might be spatially correlated, i.e. not necessarily independent.

- What weight should one give to each data set ?

The above points have been discussed in many papers in the cosmological literature and also at this conference. Here we focus on the last point. A conventional approach does not take into account the fact that different systematics may affect each data set. The problem arises when data sets are inconsistent with one another. One approach is to combine inconsistent data sets in the hope that the various systematic effects will tend to cancel out. However, this may lead to problems if all of parameter space is ruled out by one data set or another. The orthogonal approach is to choose, somewhat ad-hoc, a mutually, consistent group of data sets to combine. Lahav et al. (2000; hereafter L2000) presented a more objective method for dealing with disagreement between data sets by utilising 'Hyper Parameters' (hereafter HPs). Some previous approaches to this problem of assigning the relative weights of different measurements have been suggested in the astronomical literature (e.g. Godwin \& Lynden-Bell 1987; Press 1996).

The derivation of HPs is given in Section 2. In Section 3 we apply the method to the recent Boomerang and Maxima CMB experiments, and we estimate the best fit Hubble constant $\left(H_{0}=100 \mathrm{~h} \mathrm{~km} / \mathrm{sec} / \mathrm{Mpc}\right)$. Extensions of the methods are discussed in Section 4.

\section{2. 'Hyper-Parameters'}

Assume that we have two independent data sets, $D_{A}$ and $D_{B}$ (with $N_{A}$ and $N_{B}$ data points respectively) and that we wish to determine a vector of free parameters $\mathbf{w}$ (such as the density parameter $\Omega_{\mathrm{m}}$, the Hubble constant $H_{0}$ etc.). This is commonly done by minimising

$$
\chi_{\text {joint }}^{2}=\chi_{A}^{2}+\chi_{B}^{2},
$$

(or, more generally, maximizing the product of Likelihood functions).

Such procedures assume that the quoted observational random errors can be trusted, and that the two (or more) $\chi^{2}$ s have equal weights. However, when combining 'apples and oranges' one may wish to allow freedom in the relative weights. One possible approach is to generalise Eq. 1 to be

$$
\chi_{\text {joint }}^{2}=\alpha \chi_{A}^{2}+\beta \chi_{B}^{2},
$$

where $\alpha$ and $\beta$ are 'Hyper-Parameters', which are to be dealt with the following Bayesian way. There are a number of ways to interpret the meaning of the HPs. One way is to understand $\alpha$ and $\beta$ as controlling the relative weight of the two data sets. It is not uncommon that astronomers accept and discard measurements (e.g. by assigning $\alpha=1$ and $\beta=0$ ) in an ad-hoc way. The procedure proposed by L2000 gives an objective diagnostic as to which measurements are 
problematic and deserve further understanding of systematic or random errors. A simple example of the HPs is the case that

$$
\chi_{A}^{2}=\sum \frac{1}{\sigma_{i}^{2}}\left[x_{\mathrm{obs}, i}-x_{\mathrm{pred}, i}(\mathbf{w})\right]^{2},
$$

where the sum is over $N_{A}$ measurements and corresponding predictions and errors $\sigma_{i}$. Hence by multiplying $\chi^{2}$ by $\alpha$ we may interpret each error as effectively becoming $\alpha^{-1 / 2} \sigma_{i}$.

How do we eliminate the unknown HPs $\alpha$ and $\beta$ ? L2000 followed the Bayesian formalism given (in other contexts) in Gull (1989), MacKay (1992), Bishop (1995) and Sivia (1996). By marginalisation over $\alpha$ and $\beta$ we can write the probability for the parameters $\mathbf{w}$ given the data:

$$
P\left(\mathbf{w} \mid D_{A}, D_{B}\right)=\iint P\left(\mathbf{w}, \alpha, \beta \mid D_{A}, D_{B}\right) d \alpha d \beta .
$$

Using Bayes' theorem we can write the following relations:

$$
P\left(\mathbf{w}, \alpha, \beta \mid D_{A}, D_{B}\right)=\frac{P\left(D_{A}, D_{B} \mid \mathbf{w}, \alpha, \beta\right) P(\mathbf{w}, \alpha, \beta)}{P\left(D_{A}, D_{B}\right)},
$$

and

$$
P(\mathbf{w}, \alpha, \beta)=P(\mathbf{w} \mid \alpha, \beta) P(\alpha, \beta) .
$$

We now make the following assumptions:

$$
\begin{gathered}
P\left(D_{A}, D_{B} \mid \mathbf{w}, \alpha, \beta\right)=P\left(D_{A} \mid \mathbf{w}, \alpha\right) P\left(D_{B} \mid \mathbf{w}, \beta\right), \\
P(\mathbf{w} \mid \alpha, \beta)=\text { const. } \\
P(\alpha, \beta)=P(\alpha) P(\beta) .
\end{gathered}
$$

With the choice of 'non-informative' uniform priors in the $\log , P(\ln \alpha)=P(\ln \beta)=$ 1 we get $P(\alpha)=1 / \alpha$ and $P(\beta)=1 / \beta$ (Jeffreys 1939). Note that the integral over priors of this kind diverges (such a prior is called 'improper', see Bishop 1995). These are very conservative priors, essentially stating that we are ignorant about the scale of measurements and errors. The other extreme is obviously $P(\alpha)=\delta(\alpha-1)$, i.e. when the measurements and errors are taken faithfully. One can try other forms (see below), but it is likely that these two extreme forms reasonably bracket the probability space. Hence:

$$
P\left(\mathbf{w} \mid D_{A}, D_{B}\right)=\frac{1}{P\left(D_{A}, D_{B}\right)} P\left(D_{A} \mid \mathbf{w}\right) P\left(D_{B} \mid \mathbf{w}\right),
$$

where

$$
P\left(D_{A} \mid \mathbf{w}\right) \equiv \int P\left(D_{A} \mid \mathbf{w}, \alpha\right) \alpha^{-1} d \alpha
$$

and

$$
P\left(D_{B} \mid \mathbf{w}\right) \equiv \int P\left(D_{B} \mid \mathbf{w}, \beta\right) \beta^{-1} d \beta
$$


It is common to have a likelihood function of the form of a Gaussian in $N_{A}$ dimensions:

$$
P_{G}\left(D_{A} \mid \mathbf{w}\right) \propto \exp \left[-\chi_{A}^{2} / 2\right]
$$

where we assume for simplicity that the normalization constant is independent of the parameters $\mathbf{w}$ (this is indeed the case in our application for the CMB measurements in the next Section).

We generalise this form to incorporate $\alpha$ as follows:

$$
P\left(D_{A} \mid \mathbf{w}, \alpha\right) \propto \alpha^{N_{A} / 2} \exp \left(-\frac{\alpha}{2} \chi_{A}^{2}\right) .
$$

The integral of Eq. 11 then gives

$$
P\left(D_{A} \mid \mathbf{w}\right) \propto\left(\chi_{A}\right)^{-N_{A}},
$$

and similarly for Eq. 12. We note that it is the specific choice of prior for $P(\alpha)=1 / \alpha$ that has led to a change from a Gaussian distribution (Eq. 13) to a power-law (Eq. 15). Eq. 10 can then be written (ignoring constants) as

$$
-2 \ln P\left(\mathbf{w} \mid D_{A}, D_{B}\right)=N_{A} \ln \left(\chi_{A}^{2}\right)+N_{B} \ln \left(\chi_{B}^{2}\right) .
$$

To find the best fit parameters $\mathbf{w}$ requires us to minimise the above probability in the w space. Note that in this case our method is equivalent to assuming that we are ignorant of the relative scale of the errors in each experiment. It is as easy to calculate this statistic as the standard $\chi^{2}$. Eq. 16 actually generalises a similar equation derived by Cash (1979) using an entirely different set of assumptions.

Since $\alpha$ and $\beta$ have been eliminated from the analysis by marginalisation they do not have particular values that can be quoted. Rather, each value of $\alpha$ and $\beta$ has been considered and weighted according to the probability of the data given the model. However, it may be useful to know which values of $\alpha$ and $\beta$ were given the most weight. This can be estimated by finding the values of $\alpha$ and $\beta$ at which Eq. 14 peaks:

$$
\alpha_{\mathrm{eff}}=\frac{N_{A}}{\chi_{A}^{2}}
$$

and similarly

$$
\beta_{\text {eff }}=\frac{N_{B}}{\chi_{B}^{2}}
$$

both evaluated at the joint peak. We note that if we substitute these effective $\alpha$ and $\beta$ in Eq. 2 we obtain $\chi_{\text {joint }}^{2}=N_{A}+N_{B}$.

There is of course freedom in choosing the prior. For example, if we take $P(\alpha)=1$ (instead of Jeffreys' prior $P(\alpha)=1 / \alpha$ ) we find that the function to be minimised is

$$
-2 \ln P\left(\mathbf{w} \mid D_{A}, D_{B}\right)=\left(N_{A}+2\right) \ln \left(\chi_{A}^{2}\right)+\left(N_{B}+2\right) \ln \left(\chi_{B}^{2}\right)
$$

instead of Eq. 16. Thus these two priors give very similar results for large $N_{A}$. Numerous other priors are possible (e.g. a top-hat centred on a plausible value), but at the expense of more free HPs (e.g. the width of the top-hat). Illustrations of the HPs approach applied to toy-models are given in Bridle (2000), and another application of the above HPs (to galaxy cluster data) is given in Diego et al. (2000). 
Cosmological Parameters and Hyper-Parameters

\section{Application to the CMB Data}

\subsection{The Boomerang and Maxima Data}

The recent Boomerang (hereafter B; de Bernardis et al. 2000) and Maxima (hereafter M; Hanany et al. 2000) CMB anisotropy measurements yielded highquality angular power spectra $C_{l}$ over the spherical harmonics $400 \lesssim l \lesssim 800$. An important factor in interpreting the data is the calibration error. The experimental papers quote calibration errors of $10 \%$ and $4 \%$ (1-sigma in $\Delta T / T$ ) for $\mathrm{B}$ and $\mathrm{M}$, respectively. The measurements (with $\mathrm{B}$ data corrected upward by $10 \%$, and $\mathrm{M}$ data corrected downward by $4 \%$ ) are shown in Figure 1, and they indicate a well defined first acoustic peak at $l \sim 200$, with less convincing second and third peaks at higher harmonics. These measurements favour (under certain assumptions) a flat universe, spectral index $n=1$ and baryon density $\Omega_{b} h^{2} \sim 0.03$ (e.g. Jaffe et al. 2000; Bond et al. 2000; Bridle, this volume), which is about 2-sigma higher than the Big-Bang Nucleosynthesis (BBN) value $\Omega_{b} h^{2} \sim 0.0190 \pm 0.0018$ (95\% CL; Burles et al. 2000). Note that the recent CBI result (Padin et al. 2000) gives a higher power (at $l \sim 600$ ) relative to B\&M. Jaffe et al. (2000) fitted models after combining the B\& M data sets into one set. Here we take a different approach for joint analysis of the two data sets by utilising the 'Hyper-Parameters'.

\subsection{Results}

We illustrate the effect of using HPs by application to measurements of the angular power spectrum of the Cosmic Microwave Background (CMB). Numerous groups have now used CMB data to estimate cosmological parameters. The most common method is the flat bandpower method (Bond 1995) in which the difference between observed and predicted flat bandpowers are compared using the $\chi^{2}$ statistic (Eq. 3). We note that non-zero correlations between the CMB data points can make the data points look more smooth which, since the theoretical model is smooth on this scale, will tend to improve the apparent goodness of fit to the model and thus inappropriately give more weight to correlated data points. We also note that the assumption that the Likelihood function is a Gaussian is only an approximation (Douspis et al. 2000).

L2000 applied the HPs approach to the pre-B\&M CMB data sets, in different combinations. Here we apply the method to the recent high-quality B\&M data, first in their 'raw' form and then in their calibrated form. For simplicity, we restrict ourselves to a very limited set of cosmological models. We obtain theoretical CMB power-spectra using the CMBFAST and CAMB codes (Slejak \& Zaldarriaga 1996; Lewis, Challinor \& Lasenby 2000). We assume that CMB fluctuations arise from adiabatic initial conditions with Cold Dark Matter $(\mathrm{CDM})$ and negligible tensor component, in a flat Universe with $\Omega_{\mathrm{m}}=0.3$, $\lambda=1-\Omega_{\mathrm{m}}=0.7, n=1, Q_{\mathrm{rms}}=18 \mu \mathrm{K}$ and $\Omega_{b} h^{2}=0.03$. This choice is motivated by numerous other studies which combined CMB data with other cosmological probes (e.g. Jaffe et al. 2000, Bridle et al. 2000; Hu et al. 2000). We then investigate the constraints on the remaining parameter, the dimensionless Hubble constant, $h=H_{0} /\left(100 \mathrm{kms}^{-1} \mathrm{Mpc}^{-1}\right)$. Increasing $h$ decreases the height of the first acoustic peak, and makes few other significant changes to the angular power spectrum (e.g. Hu et al. 2000). The range in $h$ investigated here 

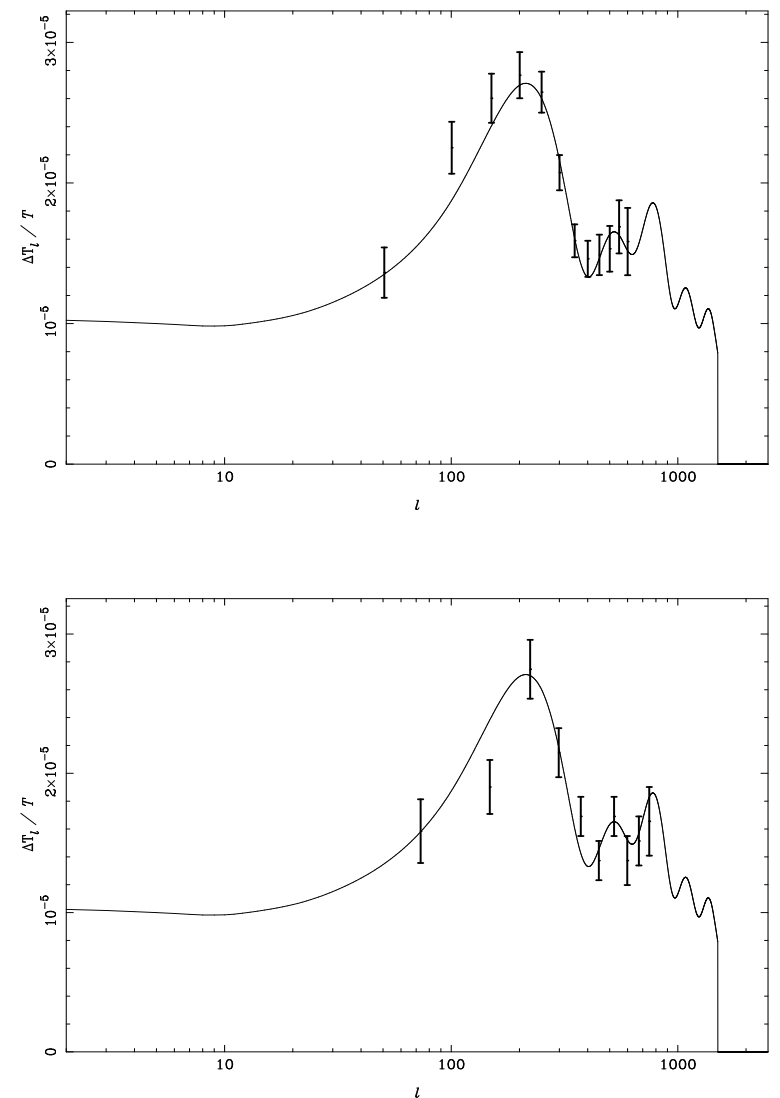

Figure 1. The Boomerang $\frac{\Delta T}{T}$ data (top panel, calibrated by 1.10) and Maxima data (bottom panel, calibrated by 0.96 ). The line in each panel is for a $\Lambda$-CDM model with $n=1, \Omega_{m}=1-\Lambda=0.3, \Omega_{b} h^{2}=$ $0.03, Q_{\mathrm{rms}}=18 \mu K$, and our 'best fit' $h=0.8$. 

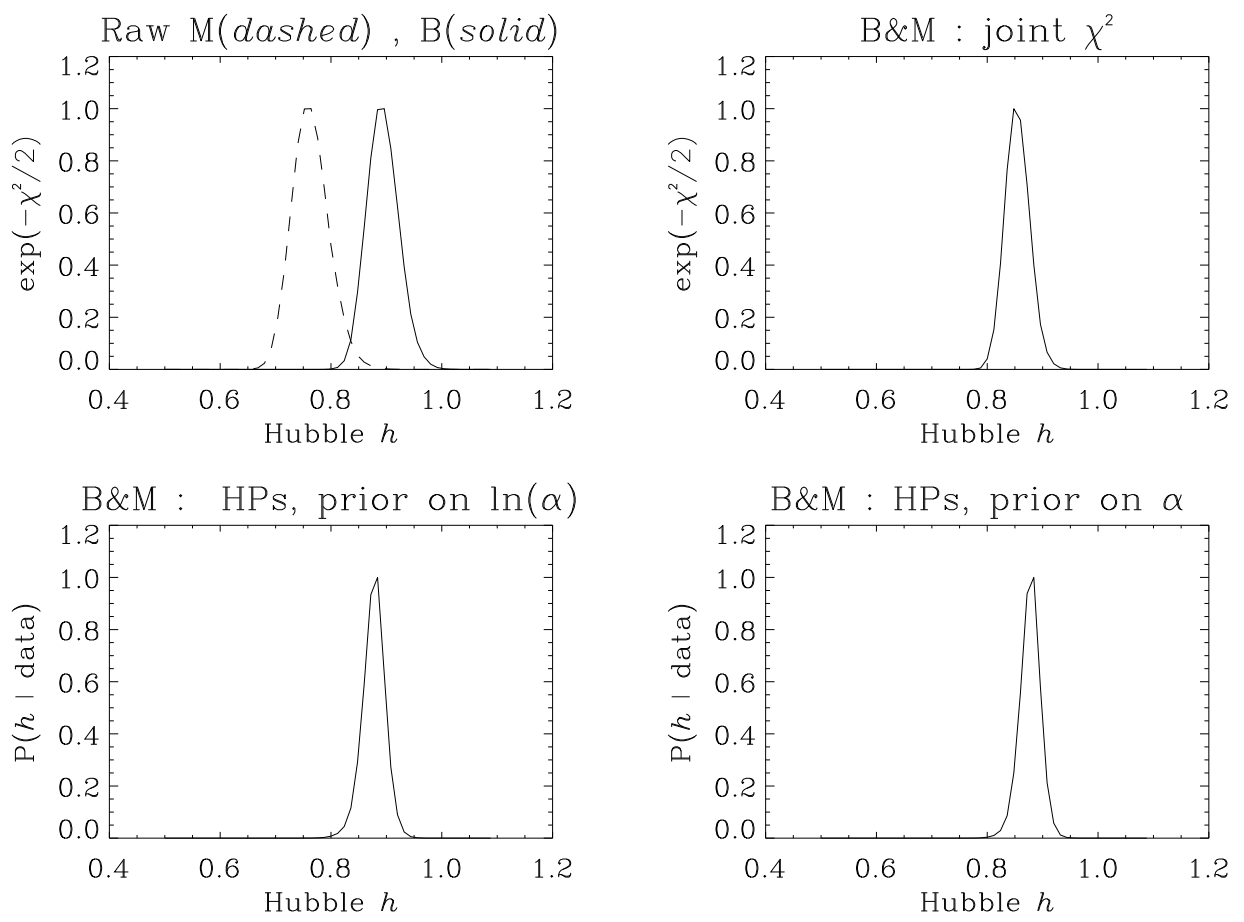

Figure 2. Analysis with raw (uncalibrated) Boomerang \& Maxima data. The top-left panel is for the individual $\chi^{2}$ of each data sets (eq. 3 ), while the top-right panel is for the sum of $\chi^{2}$ (Eq. 1). The bottomleft panel is the Hyper-Parameters probability with the prior $P(\ln \alpha)=$ 1 (Eq. 16), and the bottom left panel is for the prior $P(\alpha)=1$ (Eq. $19)$.

is $(0.5<h<1.1)$. The results using conventional $\chi^{2}$ (Eqs. 1 and 3$)$ are shown in Table 1, and with the HPs approach (Eq. 16) in Table 2. The full likelihood functions are given in Figures 2 and 3. We see that the raw (uncalibrated) B\&M data give two distinct values in the standard $\chi^{2}$ analysis. The HPs approach on the raw data suggests that $\mathrm{B}$ carries 4.5 times more weight than $\mathrm{M}$ (the ratio of the HPs), for this particular choice of model and parameter space, yielding a best $h=0.88$. However, the calibration of the data (as described in the caption to Table 1) brings the two data sets to much better agreement (e.g. the ratio of the B/M HPs is now 1.3). In fact, in this case the standard joint $\chi^{2}$ and the HPs (for two different choices of priors; Eqs. 16 and 19) give the same result, $h=0.79$, with slightly smaller error bars in the HPs case $( \pm 0.04 ; 95 \%$ CL $)$. This best fit model is shown in Figure 1. We also tried the BBN value $\Omega_{b} h^{2}=0.019$ (last entries in Table 1 and 2), which we can see gives much poorer $\chi^{2}$ than the value $\Omega_{b} h^{2}=0.03$ (as also suggested by Jaffe et al. 2000 and others). 

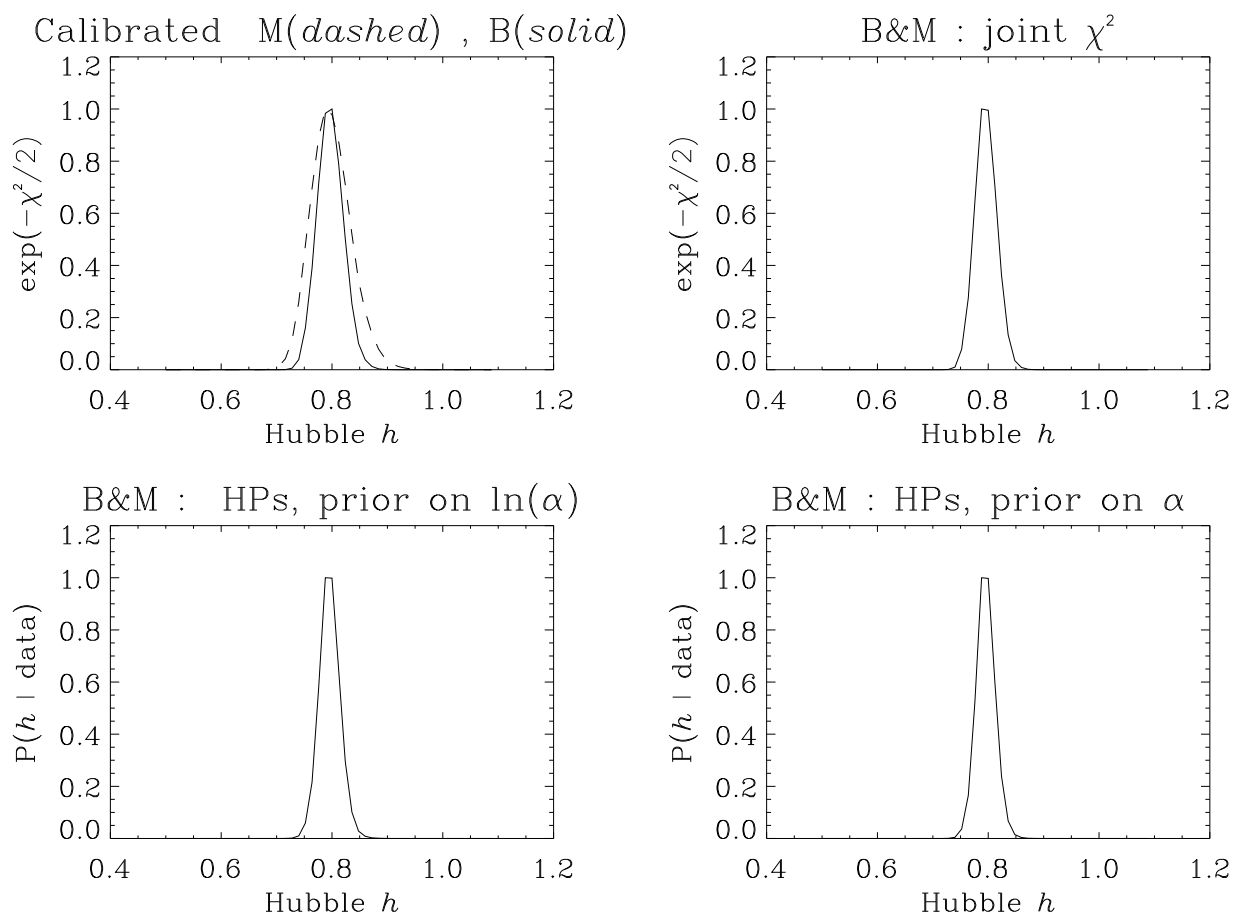

Figure 3. Analysis with calibrated B \& M data (by 1.10 and 0.96 in $\frac{\Delta T}{T}$, respectively). The panels are as in Figure 2. Note the good agreement between the two data sets, and between the joint $\chi^{2}$ approach and the HPs approach (for the two different priors).

\begin{tabular}{lrrr}
\hline Data & $N$ & Best $h$ & $\chi^{2}$ \\
\hline BOOMERANG (raw) & 12 & 0.90 & 4.4 \\
\hline MAXIMA (raw) & 10 & 0.76 & 7.1 \\
\hline B\&M (raw) & 22 & 0.85 & 19.8 \\
\hline BOOMERANG (calibrated by 1.10) & 12 & 0.80 & 8.8 \\
\hline MAXIMA (calibrated by 0.96) & 10 & 0.79 & 8.9 \\
\hline B\&M (calibrated) & 22 & 0.79 & 17.7 \\
\hline B\&M (calibrated, BBN) & 22 & 0.72 & 35.7 \\
\hline
\end{tabular}

Table 1. Conventional $\chi^{2}$ analysis using each $\mathrm{B}$ and $\mathrm{M}$ data set alone, and both sets combined. Results are given for 'raw' (uncalibrated) data, and for calibration of $\Delta T / T$ by factors of 1.10 and 0.96 for $\mathrm{B} \& \mathrm{M}$ respectively, as explained in the text. For each data set the number of data points, $N$, the best fit value of $h$ and the $\chi^{2}$ value at this point are given. The full likelihood distributions in $h$ are shown in Figures 2 and 3. Other parameters are fixed for a $\Lambda$-CDM model at $\Omega_{\mathrm{m}}=0.3, \lambda=1-\Omega_{\mathrm{m}}=0.7, n=1, Q_{\mathrm{rms}}=18 \mu \mathrm{K}$ and $\Omega_{b} h^{2}=0.03$. For the last entry $\Omega_{b} h^{2}=0.019$ (BBN value). 


\begin{tabular}{lrrl}
\hline Data & $N$ & Best $h$ & Effective HP \\
\hline B\&M (raw) & 22 & 0.88 & $2.7(\mathrm{~B}) ; 0.6(\mathrm{M})$ \\
\hline B\&M (calibrated) & 22 & 0.79 & $1.4(\mathrm{~B}) ; 1.1(\mathrm{M})$ \\
\hline B\&M (calibrated, BBN) & 22 & 0.73 & $0.5(\mathrm{~B}) ; 0.7(\mathrm{M})$ \\
\hline
\end{tabular}

Table 2. The results of the Hyper-Parameters analysis (Eq. 16) The data sets are as described in Table 1. Shown are the number of data points $N$ in each data set, the best fitting value of $h$, and the effective HP $\left(N / \chi^{2}\right)$ at this $h$. Other parameters were held fixed as described in Table 1.

\section{Discussion}

We have presented a formalism for analysing a combination of measurements, when it is likely that different systematics (or methods for calculating random errors) may affect each data set differently. By using a Bayesian analysis, and by using a specific 'non-informative' prior for the 'Hyper-Parameters' $(P(\ln \alpha)=1)$, we find that for $M$ data sets one should minimise

$$
-2 \ln P(\mathbf{w} \mid \text { data })=\sum_{j=1}^{M} N_{j} \ln \left(\chi_{j}^{2}\right),
$$

where $N_{j}$ is the number of measurements in data set $j=1, \ldots, M$. It is as easy to calculate this statistic as the standard $\chi^{2}$. The corresponding HPs $\alpha_{\text {eff }, j}=N_{j} / \chi_{j}^{2}$ provide useful diagnostics on the reliability of different data sets. We emphasize that a low HP assigned to an experiment does not necessarily mean that the experiment is 'bad', but rather it calls attention to look for systematic effects or better modelling.

In L2000 we analysed pre-B\&M data and found that while the standard $\chi^{2}$ approach gave a wide range for $H_{0}$, the Hyper-Parameter analysis suggested two distinct values of $H_{0}, \sim 50$ and $\sim 70 \mathrm{~km} / \mathrm{sec} / \mathrm{Mpc}$. Here we applied the method to the B \& M data, with and without calibration. The HPs indeed 'detect' inconsistencies between the two 'raw' data sets, but the calibrated data sets show good agreement with each other, as seen in both the $\chi^{2}$ and the HPs statistics. We have also seen in this example that the HPs solution is insensitive to the exact choice of prior.

The best fit Hubble constant is $H_{0}=79 \pm 4 \mathrm{~km} / \mathrm{sec} / \mathrm{Mpc}(95 \%$ CL, random errors only) for a fixed flat CDM $\Omega_{\mathrm{m}}=1-\lambda=0.3$ model with $n=1, Q_{\mathrm{rms}}=$ $18 \mu \mathrm{K}$ and $\Omega_{b} h^{2}=0.03$. We note that if more cosmological parameters are left free and then marginalised over, the error in $h$ would typically be larger (e.g. Bond, Bridle in this volume).

This combination of $\Omega_{m}$ and $H_{0}$ corresponds gives for the age of the Universe 11.9 Gyr. Our derived $H_{0}$ is slightly higher but still consistent with the 'final result' of $H_{0}$ from Cepheids and other distance indicators (Freedman et al. 2000) $H_{0}=72 \pm(3)_{r} \pm(7)_{s} \mathrm{~km} / \mathrm{sec} / \mathrm{Mpc}$ (1-sigma random and systematic errors).

The above analysis can be extended in a number of ways. Current and future CMB data can be combined with other cosmological probes (and their 
corresponding HPs), and more cosmological parameters can be kept free. Here we used a simple correction for the calibration error. A more general approach is to marginalise over both the HPs and a calibration probability function (Bridle et al, in preparation). Two other aspects which can be modified according to specific problems are the priors $P\left(\alpha_{j}\right)$ and the probability functions $P\left(D_{j} \mid \mathbf{w}\right)$. We shall discuss these extensions elsewhere.

Acknowledgments. I thank my collaborators S. Bridle, M. Hobson, A. Lasenby and L. Sodre for their contribution, and to L. Page and J. Mould for helpful discussions.

\section{References}

Bahcall, N.A., Ostriker, J.P., Perlmutter, S., Steinhardt, P.J., 1999, Science, 284,148

de Bernardis, P. et al., 2000, Nature, 404, 955

Bishop, C.M., 1995, Neural Networks for Pattern Recognition, Oxford University Press, Oxford

Bond, J. R., 1995, Cosmology and Large Scale Structure, Proc. Les Houches School, Session LX, August 1993. ed Schaeffer, R. Elsevier Science Publishers, Netherlands

Bond, J.R. et al., 2000, this volume (astro-ph/0011378)

Bridle, S.L., Eke, V.R., Lahav, O., Lasenby, A.N., Hobson, M.P., Cole, S., Frenk, C.S., Henry, J.P., 1999, MNRAS, 310, 565

Bridle S.L., Zehavi I., Dekel A., Lahav O., Lasenby A.N., Hobson M.P., 2000, MNRAS, in press astro-ph/0006170

Bridle S.L., 2000, PhD thesis, University of Cambridge

Burles, S., Nollett, K.M., Turner, M.S., 2000 astro-ph/0008495)

Cash, W., 1979, ApJ, 228, 939

Diego J.M. et al., 2000, submitted to MNRAS

Douspis, M., Bartlett, J.G., Blanchard, A., Dour M. Le, 2000, submitted to A\&A.

Efstathiou G., Bridle S. L., Lasenby A. N., Hobson M. P., Ellis R. S. 1999, MNRAS, 303, L47

Eisenstein, D.J., Hu, W., Tegmark, M., 1999, ApJ, 518, 2

Freedman, W.L., et al., 2000, ApJ, in press (astro-ph/0012376)

Gawiser E., Silk J., 1998, Science, 280, 1405

Godwin, P., Lynden-Bell, D. 1987, MNRAS, 229, 7

Gull, S.F., 1989, in Maximum Entropy and Bayesian Methods, Cambridge 1988, ed. J. Skilling, p. 53, Dordrecht: Kluwer

Hanany, S. et al., 2000, submitted to ApJL astro-ph/0005123)

Hu, W., Fukugita, M., Zaldarriaga, M., Tegmark, M., 2000, submitted to ApJ astro-ph/0006436)

Jaffe et al., 2000, submitted to PRL (astro-ph/0007333)

Jefferys, H., 1939, Theory of Probability, Oxford University Press (1983 edition) 
Lahav O., Bridle S.L., Hobson M.P., Lasenby A.N., Sodr'e L. Jr., 2000, MNRAS, $315,45 \mathrm{~L}$

Lewis, A., Challinor, A., Lasenby, A. 2000, ApJ, in press astro-ph/9911177)

Lineweaver, C. H. 1998, ApJ, 505, L69

MacKay, D., 1992, Neural Computation, 4, 415

Padin, S. et al, submitted to ApJ (astro-ph/0012211)

Press, W.H., 1996, in Unsolved Problems in Astrophysics, Proceedings of Conference in Honor of John Bahcall, ed. J.P. Ostriker, Princeton: Princeton University Press astro-ph/9604126)

Seljak, U., Zaldarriaga, M. 1996, ApJ, 469, 437

Sivia, D. S. 1996, Data Analysis A Bayesian Tutorial, Oxford University Press

Tegmark, M., Zaldarriage, M., 2000, preprint (astro-ph/0002091)

Webster, M., Bridle, S.L., Hobson, M.P., Lasenby, A.N., Lahav, O., \& Rocha, G. 1998, ApJ Lett, 509, L65 